\title{
Sipuleucel-T: immunotherapy for advanced prostate cancer
}

\author{
This article was published in the following Dove Press journal: \\ Open Access Journal of Urology \\ 2 May 20II \\ Number of times this article has been viewed
}

\author{
Brian M Olson \\ Douglas G McNeel \\ University of Wisconsin Carbone \\ Cancer Center, University of \\ Wisconsin-Madison, Madison, WI, USA
}

\begin{abstract}
Prostate cancer continues to be one of the most serious afflictions of men of advanced age, remaining the most commonly diagnosed and second leading cause of cancer-related deaths in American men. The treatment options for patients with incurable metastatic, castrate-resistant disease have long focused on various chemotherapeutic approaches, which provide a slight survival benefit while being associated with potentially significant side effects. However, the recent approval of sipuleucel-T has given patients with advanced disease an additional treatment option that has demonstrated benefit without the side effects associated with chemotherapy. Sipuleucel-T is an antigen-presenting cell-based active immunotherapy that utilizes a patient's own immune cells, presumably to activate an antigen-specific immune response against tumor cells. This review focuses on the development and implementation of sipuleucel-T as a therapy for prostate cancer. Specifically, we present some of the issues associated with the management of advanced prostate cancer, the research and development that led to the approval of sipuleucel-T, how the approval of sipuleucel-T could change the clinical management of prostate cancer, and current and future areas of investigation that are being pursued with regard to sipuleucel-T and other treatments for advanced prostate cancer.
\end{abstract}

Keywords: sipuleucel-T, prostatic acid phosphatase, granulocyte-macrophage colonystimulating factor

\section{Management of advanced prostate cancer}

Prostate cancer is one of the most widespread afflictions in men of advanced age, remaining the most commonly diagnosed and second leading cause of cancer-related death in American men. ${ }^{1}$ Most of these tumors are diagnosed as organ-confined, androgen-dependent malignancies, and are commonly treated by surgical removal of the prostate or by radiation therapy. This treatment is curative for the majority of patients, but approximately one-third of patients will experience biochemical recurrence of disease, as measured by increases in the serum prostate-specific antigen (PSA) marker, within 10 years. $^{2-4}$ Ultimately this will lead to radiographically detectable metastases, most often to sites in bone and lymph nodes which is typically treated with androgen deprivation. Patients who develop metastatic disease have a median survival of 3-7 years. ${ }^{2,4}$ Unfortunately, following androgen deprivation the disease typically recurs as castrate-resistant prostate cancer, which is the lethal phenotype of the disease. ${ }^{4-6}$ Castrate-resistant, metastatic prostate cancer is incurable, with patients eventually succumbing to their disease after approximately 12-24 months.

Mitoxantrone (a chemotherapeutic agent that inhibits type II topoisomerase and results in disrupted DNA synthesis and repair) was the first therapy approved by the
Correspondence: Douglas G McNeel 7007 Wisconsin Institutes for Medical Research, IIII Highland Avenue, Madison, WI 53705, USA

$\mathrm{Tel}+|608265813|$

Fax + I 6082650614

Emaildm3@medicine.wisc.edu
Open Access Journal of Urology 201 I:3 49-60 
US Food and Drug Administration (FDA) for the treatment of patients with castrate-resistant, metastatic disease. However, mitoxantrone was approved based on its ability to reduce the pain associated with bone metastasis and its ability to improve the quality of life of men with advanced disease, but it was not found to prolong overall survival in men receiving the drug versus those receiving best supportive care. $^{7-9}$ It was not until the development of the taxanes, a class of chemotherapeutic agents targeting microtubule formation, that any chemotherapeutic regimen was clearly demonstrated to provide a survival benefit to patients with metastatic, castrate-resistant disease. In 2004, the results of two Phase III trials evaluating the taxane docetaxel, showed that patients receiving this chemotherapy had a median survival of slightly more than two months longer than those receiving mitoxantrone. ${ }^{10,11}$ This led docetaxel to become the first-line standard of care for patients with symptomatic, castrate-resistant, metastatic disease.

Until 2010, there were no standard second-line treatments for patients whose disease had progressed following docetaxel treatment, although mitoxantrone, with its demonstration of disease palliation, was often used. Cabazitaxel (another of the taxane class of chemotherapy) was recently FDA-approved in this setting following the results of a large randomized trial demonstrating a survival benefit of approximately 2.4 months over those treated with mitoxantrone. ${ }^{12}$ Of note, the mortality rate within 30 days of treatment with cabazitaxel was $5 \%$, mostly attributed to neutropenia and its consequences, underscoring the potential toxicities of chemotherapy agents in this stage of disease. ${ }^{12}$ Many other agents are in development, including novel androgen synthesis inhibitors (such as abiraterone and TAK-700) and androgen receptor antagonists (such as MDV-3100). At the 2010 meeting of the European Society for Medical Oncology, the interim results of a Phase III trial evaluating abiraterone (a CYP17 inhibitor) versus prednisone were released. ${ }^{13}$ In the same setting of patients with docetaxel-resistant, castrate-resistant, metastatic prostate cancer, this agent demonstrated an improvement in overall survival of over four months, as well as superior outcomes for time to progression and disease response. The trial was closed early, and FDA approval is currently being sought. The development, and possible approval, of agents such as abiraterone will certainly challenge the use of agents with greater toxicity, such as cabazitaxel, and will present new challenges with regard to the timing and sequencing of all available treatments for patients with advanced disease.

\section{Prostate cancer immunotherapy}

The ability of the immune system to recognize and eliminate tumor cells was first recognized more than a century ago by Paul Ehrlich, and later revived by the work of Thomas and Burnet half a century later. ${ }^{14-16}$ While their "immune surveillance hypothesis" has undergone considerable evolution as our understanding of the immune system and cancer biology has grown, research has shown that the immune system has the capability to recognize and possibly protect individuals from tumor growth. This immune recognition of tumors is often manifested by pre-existing immune responses in patients with cancer to particular tumor antigens expressed by tumor cells, enhanced prognosis and overall survival of patients with tumors that are highly infiltrated by lymphocytes, and in some exceptionally rare cases, spontaneous tumor regression mediated by the immune system. ${ }^{17-25}$

The prostate has been shown to be a potentially immunogenic tissue, being predisposed to prostatitis, a syndrome of pelvic pain which has been suggested to be in part mediated by an autoimmune response to prostate tissue antigens. ${ }^{26,27}$ Furthermore, this immunologic recognition of the prostate is also found in the context of malignant disease. For example, one of the earliest premalignant lesions of the prostate, (ie, proliferative inflammatory atrophy), is characterized by lymphocytic infiltrates. ${ }^{28}$ Moreover, tumor-infiltrating lymphocytes can be detected in prostate tumors, and increased tumor-infiltrating lymphocytes have been shown to correlate with a decreased rate of prostate cancer recurrence. ${ }^{25}$ Work has also shown that patients with prostate cancer commonly have pre-existing immune responses to many different prostate antigens, such as PSA, ${ }^{22}$ prostatic acid phosphatase (PAP), ${ }^{22,29,30}$ prostatespecific membrane antigen, ${ }^{30}$ the androgen receptor, ${ }^{31}$ and several others. ${ }^{18,32}$ Additionally, prostate-specific immune responses can actually be enhanced by current prostate cancer therapies, including androgen deprivation therapy, which has been shown to induce prostate-specific infiltration of activated $\mathrm{T}$ cells and other immune cells, as well as enhancing prostate-directed immunotherapies. ${ }^{33-36}$ In addition, because prostate cancer has a long natural history, often with more than a decade between initial diagnosis and end-stage disease, there is a large window of time for potential immunotherapeutic intervention, leading several groups to become interested in harnessing prostate tumor immunity and focusing on it to generate an antitumor immune response. 
The principle of cancer vaccines is to use an antigencontaining vector (or the antigen itself) to activate an immune response against protein epitope/s expressed and displayed by tumor cells. These vaccines predominantly focus on activating CD8+ T cells, which have the capability to recognize and lyse tumor cells directly. To activate these CD8+T cells efficiently, tumor cell-derived epitopes must be presented by major histocompatibility complex molecules on the surface of antigen-presenting cells. Some vaccines rely on delivering the actual antigen of interest to patients, such as in the form of a recombinant protein or peptide. An example of these types of vaccines include peptide vaccines targeting PSA or multiple peptides based on an individual's peptide-specific reactivity, or protein vaccines such as those targeting NY-ESO-1.,37-39 An alternative to delivering the antigen itself is to use a vector to induce expression of the antigen in the host, such as genetic vaccines using viruses, bacteria, or DNA alone encoding the antigen. ${ }^{40-43}$ These vectors introduce the antigen to the host, where endogenous antigen-presenting cells can take up the vector and drive expression of the encoded antigen and display antigen-derived epitopes to T cells. An example of one such vaccine is Prostvac ${ }^{\mathrm{TM}}$, a vaccinia-based and fowlpoxbased vaccine that targets PSA. The Prostvac vaccine recently completed a Phase II clinical trial in men with minimally symptomatic metastatic, castrate-resistant prostate cancer, in which Prostvac was found not to meet its primary endpoint of an increase in time to disease progression, but did show an increase in overall survival of approximately 8.5 months compared with the placebo arm. ${ }^{44}$

An alternative approach to targeting one particular antigen is to target any and all antigens displayed by tumor cells, which is the approach taken by antigen-nonspecific vaccines. An example of an antigen-nonspecific vaccine for prostate cancer is $\mathrm{GVAX}^{\circledR}$, which is composed of two irradiated prostate cancer cell lines that have been genetically modified to secrete granulocyte-macrophage colony-stimulating factor (GM-CSF), an immunostimulatory cytokine. ${ }^{45,46}$ This vaccine was evaluated in two randomized Phase III clinical trials, the first (VITAL 1) evaluating GVAX versus docetaxel alone and the second (VITAL 2) studying the combination of GVAX and docetaxel versus docetaxel alone. However, before these trials could be completed, the Independent Data Monitoring Committee conducted a routine safety review of the VITAL 2 trial, which found that patients receiving GVAX and docetaxel had a higher rate of death than those receiving docetaxel alone. ${ }^{47,48}$ Based on these results, the VITAL 2 trial was closed, and a subsequent futility analysis of the VITAL 1 trial led to its closure as well.

Although both GVAX and Prostvac have been studied in advanced, randomized clinical trials, both vaccines require additional processing of the vector once delivered to the host. For CD8+ T-cell activation, vaccines such as Prostvac rely on host antigen-presenting cells taking up the viral vector and expressing the encoded antigen, or via cross-presentation by infected bystander cells. An alternative approach to activate CD8+ T cells more directly is to deliver antigen-presenting cells that have been preloaded with a tumor antigen. Early studies in prostate cancer focused on delivering antigenpresenting cells that had been pulsed with peptides derived from prostate-specific membrane antigen, an approach that showed some promise in early clinical trials in patients with castrate-resistant, metastatic disease. ${ }^{9,49}$ While this specific vaccine was not pursued in later-stage clinical trials, it helped set the stage for studies evaluating sipuleucel-T (Provenge ${ }^{\circledR}$, Dendreon Corporation, Seattle, WA), an antigen-presenting cell-based active immunotherapy targeting PAP.

\section{Sipuleucel-T: preclinical and clinical evaluation}

Sipuleucel-T is an antigen-specific vaccine targeting PAP, an antigen expressed by nearly all (about 95\%) of prostate tumors. PAP is commonly recognized in prostate cancer patients, and T cells specific for PAP have been shown to have the ability to lyse prostate tumor cells. ${ }^{10,50}$ Furthermore, PAP has been targeted using several different vaccine approaches, including DNA vaccines and peptide vaccines. ${ }^{42,51-55}$ Sipuleucel-T is an autologous antigen-presenting cell-based active immunotherapy, composed of autologous peripheral blood mononuclear cells isolated from prostate cancer patients that are cultured for 36-44 hours with PA2024, a recombinant fusion protein composed of PAP linked to GMCSF. Following culture with this fusion protein, these cells are then infused intravenously into the same patient. ${ }^{56}$ The proposed mechanism of action is that these PA2024-pulsed antigen-presenting cells then present PAP-derived epitopes to the patient's immune system, which activate PAP-specific cytolytic T-cell immune responses that can recognize and lyse prostate tumor cells.

PAP-GM-CSF-loaded antigen-presenting cell vaccines were first studied in the Copenhagen rat, given that rat PAP is nearly $80 \%$ homologous with human PAP. In this study, the authors evaluated several PAP-based immunotherapies, ie, PAP protein alone, the PAP-GM-CSF fusion protein, 
dendritic cells pulsed with either PAP or the PAP-GM-CSF fusion protein, or a combination of the PAP-GM-CSF fusion protein given along with the dendritic cell fusion proteinpulsed vaccine. In these studies, they found that while the two protein approaches were effective at inducing antibody responses, only the delivery of the PAP-GM-CSF-pulsed dendritic cell vaccine-induced prostatitis, which the authors used as a measure of "tumoricidal potency" ${ }^{57}$ Furthermore, they found that CD4+ T cells (and not CD8+ T cells) from immunized animals could inhibit the proliferation of syngeneic prostate tumor cells. ${ }^{57}$ While these preclinical studies did not evaluate the ability of this immunotherapy approach to alter tumor growth in vivo, they did provide the rationale for evaluating this therapy in a Phase I clinical trial.

Sipuleucel-T was evaluated in three separate singleagent Phase I trials in patients with metastatic, castrateresistant prostate cancer (the design and results from all the sipuleucel-T single-agent trials are summarized in Table 1). In a Phase I/II trial, Small et al treated patients with castrateresistant disease with sipuleucel-T at weeks 0,4 , and 8 , with the possibility of a fourth booster immunization. The Phase I portion of this study was conducted using a standard dose escalation, with cohorts of three patients receiving $0.2 \times 10^{9}, 0.6 \times 10^{9}, 1.2 \times 10^{9}$, and $2 \times 10^{9} \mathrm{PA} 2024$-pulsed cells per injection, and 19 patients in the Phase II portion of this trial received the maximum dose that could be prepared. For the production and delivery of the vaccine, patients underwent a leukapheresis, which was then shipped to the Dendreon cell processing facility where dendritic cell precursors were isolated using two sequential buoyant density centrifugation steps. Cells were washed and incubated with the PA2024 fusion protein for approximately 40 hours. These products were then transported back to the infusion center, where they were infused into the patients over approximately 30 minutes. In this study, all of the patients were found to develop a T-cell proliferative response to PA2024, while 38\% were found to develop responses to native PAP. ${ }^{58}$ They also identified that most patients developed immunity to PA2024 after two infusions, with a maximal reactivity after three infusions (the additional booster immunization did not confer additional immunity). Furthermore, six patients had PSA decreases greater than $25 \%$ (three of whom had a decline of greater than $50 \%$ ), and in the Phase II trial, the median time to disease progression was 29 weeks. ${ }^{58}$ Interestingly, patients who were found to develop an immune response (either T-cell and/or antibody responses) to the native PAP had a significantly longer time to disease progression than those without an immune response (34 weeks versus 13 weeks). ${ }^{58}$
In another Phase I trial evaluating sipuleucel-T in patients with metastatic, castrate-resistant disease, Takaue et al immunized patients three times at weeks 0,2 , and 4 using increasing doses of sipuleucel-T. In this study, they identified T-cell proliferative immune responses to PA2024 in all patients, and one of the patients had an objective clinical response of lymph node metastases, as well as a decrease in PSA levels. ${ }^{59}$

The last Phase I/II trial of sipuleucel-T was conducted by Burch et al. In the Phase I portion of this study, 13 patients received sipuleucel-T infusions at weeks 0 and 4 (as many cells as could be prepared from the preinfusion leukapheresis). Following these infusions, patients received subcutaneous doses of the PA2024 fusion protein at weeks 8, 12, and 16. In this study, of the 12 patients evaluable for response to treatment, they identified T-cell proliferative and antibody responses to PA2024 in all patients. When they evaluated responses against the two components of the fusion protein, they detected T-cell proliferation against both GM-CSF and PAP, but found higher levels of proliferation in response to stimulation with GM$\mathrm{CSF}^{60}$ Furthermore, the authors identified PSA decreases of greater than $50 \%$ in three patients. ${ }^{60}$ In the Phase II portion of this study, 21 patients with metastatic, castrate-resistant disease were given two infusions of sipuleucel-T at weeks 0 and 2. Patients for whom disease had not progressed (a total of 19 patients) were then subsequently boosted with the PA2024 fusion protein, given subcutaneously at weeks 4, 8, and 12. Again, they observed immune responses to PA2024 following immunization and PSA decreases in three patients, with one of these patients having an objective response that persisted for more than four years. ${ }^{61}$ The combined data from these three Phase I/II clinical trials served as the foundation for randomized Phase III trials evaluating sipuleucel-T in patients with castrate-resistant disease.

In the first Phase III trial evaluating sipuleucel-T (D9901), Small et al studied 127 men with asymptomatic, metastatic, castrate-resistant disease expressing PAP. Patients were randomly assigned in a 2:1 ratio to receive three biweekly infusions of sipuleucel-T or placebo. Patients in the sipuleucel-T arm received as many cells as could be prepared from the leukapheresis product. ${ }^{62}$ The patients receiving placebo underwent a leukapheresis, and one-third of the cells were reinfused without being pulsed with PA2024. The remaining cells were frozen, and if a patient showed disease progression, these cells could be pulsed with PA2024 and infused into the patient in an open-label salvage protocol. In this study, the primary endpoint was chosen as time to disease progression (either radiographic or development of pain), and the authors also followed overall survival 


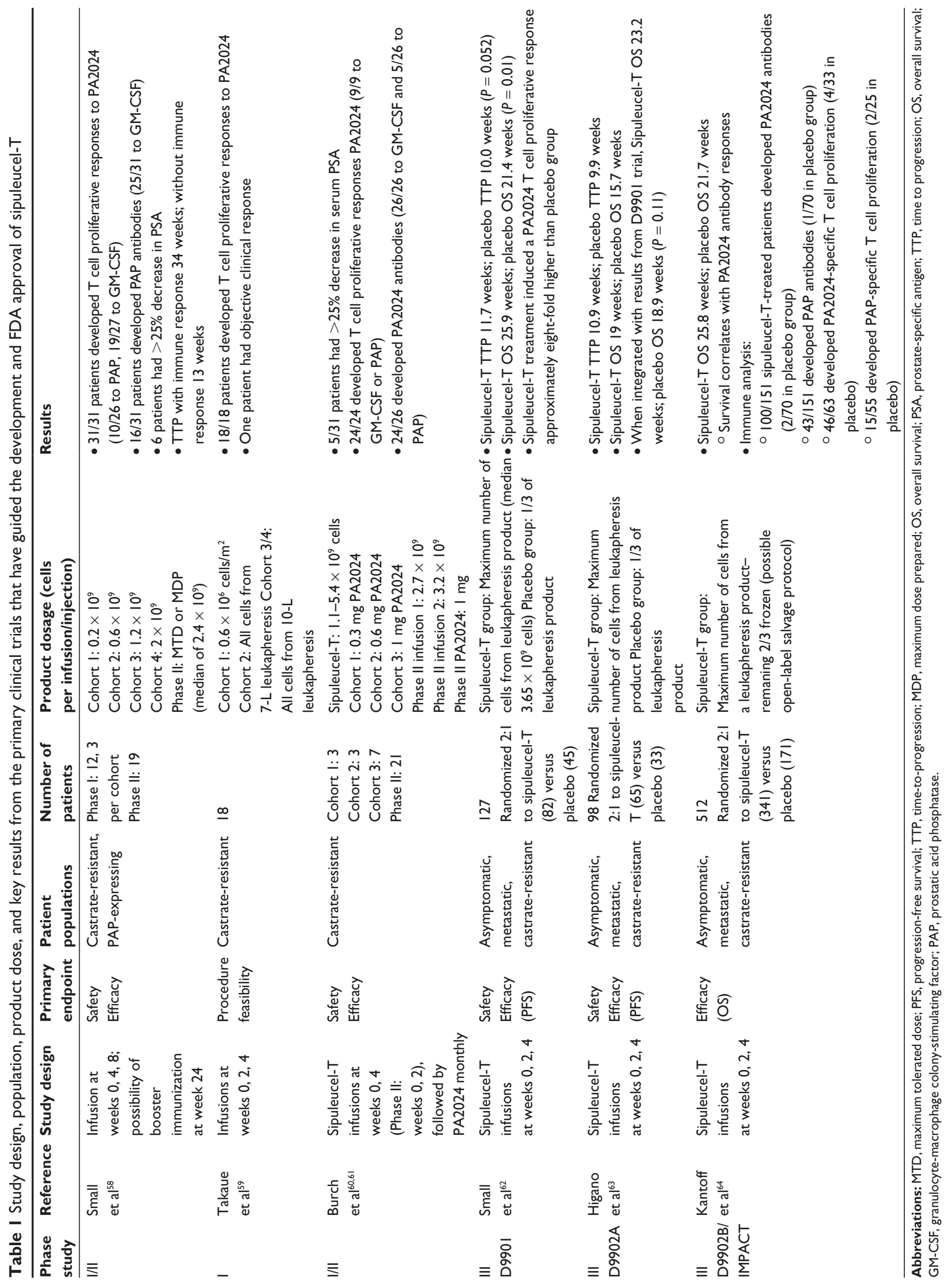


(although this was not a predetermined primary or secondary endpoint, because the trial was not sufficiently powered to detect significant differences in survival).

In this study, treatment with sipuleucel-T was found to be well tolerated by patients. The most common side effects associated with sipuleucel-T infusion were Grade 1 or 2 rigors and pyrexia $(54.9 \%$ and $37.8 \%$ of patients, respectively), with a lower frequency of patients having Grade 1 or 2 tremors (26.8\%). ${ }^{62}$ The authors also detected $\mathrm{T}$ cell proliferative responses to the PA2024 fusion protein. When they calculated the increase in the T-cell stimulation index four weeks following the last treatment compared with baseline, they found that patients receiving sipuleucel-T had an approximately eight-fold higher T-cell stimulation index than patients receiving placebo. ${ }^{62}$ However, while the authors detected serum PSA decreases of greater than $25 \%$ in $6.8 \%$ of patients receiving sipuleucel-T, the primary endpoint of time to disease progression did not reach statistical significance. Patients receiving sipuleucel-T had a median time to disease progression of 11.7 weeks (95\% confidence interval [CI] 9.1-16.6) compared with 10.0 weeks for patients receiving placebo $(95 \% \mathrm{CI}$ $8.7-13.1){ }^{62}$

While the D9901 trial was ongoing, a second companion Phase III trial was also underway, accruing patients with asymptomatic metastatic, castrate-resistant disease (D9902A). This trial was being conducted with the same parameters as in the D9901 trial, ie, a 2:1 randomization of patients to receive sipuleucel-T (given as three biweekly infusions) or placebo (given as three biweekly infusions of untreated autologous peripheral blood mononuclear cells). This trial was designed as a second, confirmatory study to D9901, with the same primary endpoint of evaluating the effect of sipuleucel-T on time to disease progression. However, while this trial was still accruing patients, the results from the D9901 trial showing a lack of difference in time to disease progression were unblinded, and due to lack of significance in the primary endpoint of time to disease progression, the D9902A trial was abandoned.

A further subset analysis of D9901 found that patients with a Gleason score $\leq 7$ treated with sipuleucel-T had a significant increase in time to disease progression compared with those receiving placebo. This led to another Phase III trial (D9902B), which was designed similarly to the D9901 trial, with 127 patients and a primary endpoint of time to disease progression, but with the plan only to accrue asymptomatic metastatic, castrate-resistant patients with a Gleason score of $\leq 7$. However, as this trial was beginning to accrue, an analysis of the D9901 trial uncovered a significant difference in overall survival, with patients receiving sipuleucel-T having a three-year overall survival of 25.9 months, compared with 21.4 months, for patients receiving placebo (hazard ratio [HR] 1.43, $P=0.01){ }^{63}$ Additionally, it was observed that nearly half of the progression events occurred within the first 12 weeks of the study, which the authors postulated might be before an optimal immunologic effect had occurred, and therefore could account for a lack of difference in time to disease progression. ${ }^{62,63}$ Furthermore, when an integrated analysis of the D9901 and D9902A trials was conducted looking at a total of 147 patients treated with sipuleucel-T versus 78 treated with placebo, it was observed that patients receiving sipuleucel-T had a median survival of 23.2 months $(95 \% \mathrm{CI}$ 19.0-31.0) versus 18.9 months (95\% CI 13.5-25.3) in the placebo control group (HR 1.5, $P=0.011) .{ }^{63}$ Due to these findings, the authors implemented an amendment to the D9902B protocol (termed the Immunotherapy for Prostate Adenocarcinoma Treatment [IMPACT] trial), changing the primary endpoint from time to disease progression to overall survival, and increasing the planned patient enrollment from 127 to 512 (with no exclusion based on Gleason score) to power this study sufficiently to detect a difference in overall survival.

The IMPACT trial confirmed that treatment with sipuleucel-T provided a significant increase in overall survival. Patients receiving an infusion of PA2024-pulsed cells had a median survival of 25.8 months compared with 21.7 months in the placebo group, consistent with the previous trials. ${ }^{64}$ This survival difference reflected a significant difference in the risk of death in patients treated with sipuleucel-T compared with the placebo group (HR 0.78, 95\% CI $0.61-0.96, P=0.03) .{ }^{64}$ The authors also found that this survival benefit was present regardless of prior or subsequent treatment with docetaxel and prednisone, ie, the standard chemotherapeutic regimen for patients with metastatic, castrate-resistant disease (although the percentage of patients receiving prior chemotherapy was relatively small). When the median time to progression was calculated, they found no significant difference (14.6 weeks in the sipuleucel-T group versus 14.4 weeks in the placebo group, $P=0.63$ ), contradicting the trends observed in the earlier-phase studies, but validating the authors' decision to focus on overall survival as a primary endpoint. ${ }^{64}$

Patients in the IMPACT trial were evaluated for the development of immune responses (either antibody or T-cell proliferative responses) to PA2024 or native PAP. 
Antibody responses were defined as a titer greater than 400 at any time after baseline, and T-cell proliferative responses were defined by a T-cell stimulation index $>5$ at any time following immunization (Dr Nadeem Sheikh, Dendreon Corporation, 2011; Pers comm) ${ }^{64}$ In patients receiving sipuleucel-T, antibody responses to PA2024 were detected in 100/151 patients, and responses to PAP were detected in 43/151 individuals (with 2/70 and 1/70 placebo patients having responses to PA2024 or PAP, respectively).$^{64}$ Furthermore, in patients receiving sipuleucel-T, proliferative T-cell responses to PA2024 were detected in 46/73 patients, and responses to PAP were detected in $15 / 55$ patients (with $4 / 33$ and $2 / 25$ patients receiving placebo having responses to PA2024 or PAP, respectively). ${ }^{64}$ It was observed that patients who had antibody responses to PA2024 lived significantly longer than those patients without antibody responses $(P<0.001)$. In contrast with what had been observed in earlier-phase studies, no significant correlation was found between the detection of T-cell proliferative responses to either PA2024 or PAP and the overall survival of these patients (nor antibodies to PAP, although this showed a trend towards correlation with survival, $P=0.08$ ).

Similar to previous trials, treatment with sipuleucel-T was most commonly associated with chills (54.1\% of patients), fever (29.3\%), fatigue (39.1\%), nausea (28.1\%), and headache (16.0\%). ${ }^{64}$ Additionally, treatment with sipuleucel-T was also associated with increased frequencies of influenza-like illness, myalgia, hypertension, hyperhidrosis, and groin pain. ${ }^{64}$ Most adverse events were graded as mild to moderate, and most occurred within one day after infusion and resolved within 1-2 days. Adverse events $\geq$ Grade 3 of any type were reported by $31.7 \%$ of patients receiving sipuleucel-T, and were not significantly different from the $35.1 \%$ of patients in the placebo group. ${ }^{64}$ However, adverse events $\geq$ Grade 3 on the day immediately following infusion were detected in 23 of $338(6.8 \%)$ patients receiving sipuleucel-T compared with three of $168(1.8 \%)$ patients in the placebo group. ${ }^{64}$ Only three of $338(0.9 \%)$ patients in the sipuleucel-T group were not able to receive all three infusions due to infusion-related adverse events.

After the initial D9901 trial suggested a survival benefit in patients treated with sipuleucel-T, Dendreon submitted a biological license application to the FDA in 2006. However, while this application was viewed favorably by an advisory committee evaluating cellular, tissue, and gene therapies, the FDA Center for Biologics Evaluation and Research requested additional data on the efficacy of sipuleucel-T before approval. With the significant survival benefit detected in the
Phase III IMPACT trial, the FDA approved sipuleucel-T in April 2010 for the treatment of asymptomatic or minimally symptomatic metastatic, castrate-resistant prostate cancer.

\section{Incorporation of sipuleucel-T into clinical practice}

The approval of sipuleucel-T for metastatic, castrate-resistant prostate cancer represents a major paradigm shift in the treatment of advanced disease. Not only does it represent the first active immunotherapy approved for cancer, but it is novel compared with other prostate cancer therapies in that there is no measurable readout that can be used to monitor the success or failure of therapy. Moreover, compared with therapies such as androgen deprivation therapy or chemotherapy, the overall survival benefit associated with sipuleucel-T is not associated with a decrease in PSA levels, objective clinical responses, or an increased time to disease progression. This presents a challenge for the implementation of sipuleucel-T in clinical practice, because it does not allow for any short-term measure of whether the therapy is providing a clinical benefit. The concept of using treatments without measurable benefit is not new to oncology or the treatment of prostate cancer; this is expected in the adjuvant setting where treatment is administered to reduce the risk of disease recurrence, or with the use of bisphosphonates to minimize the risk of skeletal events. However, in the setting of metastatic disease treatment, this does represent a new paradigm and presents a challenge for a few reasons. First, is it possible that an individual did or did not "respond" and/or might need a reinduction course to guarantee or prolong this response? Second, when should other treatments be considered? Docetaxel, for example, is typically given with corticosteroids, which are agents known to have immunosuppressive effects that may mitigate any benefit associated with sipuleucel-T. As such, a question that may commonly arise in the management of patients receiving sipuleucel-T is how long should one wait before administering immunosuppressive therapy that might negate what are perceived to be delayed effects from this vaccine? Third, what percentage of patients treated with sipuleucel-T actually "respond" to this treatment? Whether for this treatment or other costly personalized therapies, payers may ultimately demand markers predictive of response to know whether a treatment is likely to work for an individual, rather than potentially authorizing treatment of the majority to benefit a minority.

The findings from the Phase III trials also raise questions about the mechanism of action of sipuleucel-T. In fact, the package insert for sipuleucel-T states that the precise 
mechanism of action remains unknown. The original concept in loading antigen-presenting cells with the PAP-GM-CSF conjugate protein was to prime antigen-presenting cells more directly to present PAP-derived epitopes and elicit a cytolytic T-cell response specific for PAP-expressing tumor cells. ${ }^{54,58}$ To date, there have been no data in either preclinical models or clinical trials showing that this treatment elicits PAP-specific cytolytic T cells, as has been shown for other PAP-directed vaccines. ${ }^{50,51,55}$ In early-stage clinical trials, Small et al found that patients who developed either a T-cell or antibody responses to native PAP had a significantly longer time to disease progression than patients without a PAP-specific immune response, whereas in the Phase III trial, they identified that only patients with antibody responses to PA2024 had a significant increase in overall survival. ${ }^{58,64}$ It is unclear how antibodies to an intracellular or secreted antigen would contribute to an antitumor response unless they were surrogates for a cellular response. Also, more responses were identified to the PA2024 antigen than to the native PAP protein suggesting that the majority of the responses elicited were directed to the GM-CSF portion of the fusion protein. In fact, two separate Phase I/II studies showed that more patients developed immune responses to GM-CSF alone than to PAP alone. ${ }^{58,60,61}$ Therefore, to date, there remains a lack of a clear mechanism of action associating clinical benefit with the activation of PAP-specific T-cell responses in vivo. One could argue that if a therapy is safe and prolongs survival, understanding its precise mechanism of action is less important. However, without a clear biological marker of response, it will be difficult to edit this therapy (evaluating different schedules, evaluating "booster" immunization courses, or combining it rationally with other therapies to enhance its efficacy) without conducting large, long-term trials powered for overall survival.

An additional question regarding the mechanism of action of sipuleucel-T arose from the design of the placebo groups in the Phase III trials. In the D9901, D9902, and IMPACT trials, the placebo groups were infused with untreated, autologous peripheral blood mononuclear cells. While this placebo group would be expected to be safe and would control for any effects of leukapheresis and infusions on disease progression and immune responses, it does not address the issue of antigen-specificity. An alternative control would have been to treat patients on the placebo arm with peripheral blood mononuclear cells that had been pulsed only with GM-CSF. By studying the contribution that GM-CSF has towards the effects of sipuleucel-T, and thus also evaluating the antigen-specificity of this approach, it would be possible to disregard any potential contribution that other cell types present in the infusion product (including $\mathrm{B}$ cells, T cells, and natural killer cells) may have towards the efficacy of this approach. If antigen-specificity is not the central mediator of the clinical efficacy of sipuleucel-T, then this therapy could potentially be tailored to a much less expensive activated cellular therapy, or alternatively that sipuleucel-T might be investigated for other non-PAPexpressing malignancies.

While the safety and paucity of adverse events associated with sipuleucel-T are certainly advantages recognized by patients and treating physicians, a significant challenge in its widespread implementation into clinical practice is the cost associated with this therapy. Because this is an autologous cellular-based vaccine, it requires a rigorous production protocol that needs to be conducted independently for each patient and each treatment course. For each infusion cycle, the patient must first undergo a leukapheresis. This product is then sent to a central processing facility, a time-sensitive step which could make implementation of sipuleucel-T in rural clinics logistically difficult. These cells are treated with the fusion protein in a controlled setting before they are then shipped back to the clinic, where the patient is reinfused with the antigen-pulsed product. At a cost of at least $\$ 93,000$ per total six-week course, and the reimbursement policy of Medicare, Medicaid, and other third-party payers currently pending, this could further limit community implementation. In fact, while several insurance companies (including Aetna, Humana, Emblem Health, and Kaiser Permanente) have agreed to cover the costs associated with sipuleucel-T, a survey conducted in July 2010 of medical and pharmacy directors by Reimbursement Intelligence found that $65 \%$ indicated that they may restrict access to sipuleucel-T. ${ }^{65}$ As highlighted by D Longo in an editorial to the reporting of the Phase III IMPACT trial, a formal cost-utility analysis of sipuleucel-T is needed to determine if the cost of sipuleucel- $T$ per quality-adjusted life-year is in line with other cancer therapies. ${ }^{66}$ This is especially important when considering the development of Prostvac, a potentially "off-the-shelf", nonpersonalized vaccine. The Phase II trial evaluating Prostvac found that this vaccine, while not meeting its primary endpoint of increased time to disease progression, similarly showed trends towards prolonged overall survival in the same patient population in which sipuleucel-T was approved, and a Phase III trial evaluating this vaccine is expected to open in 2011. If similar results are found in this trial, it is conceivable that Prostvac could be approved for the same population of patients in the next several years, providing a potentially 
less costly alternative that would compete directly with sipuleucel-T. Nonetheless, at the present time, even with the significant manufacturing cost of this agent, one must balance the value of this novel therapy in the context of expensive chemotherapy agents that might actually provide less benefit and have greater potential toxicity.

While sipuleucel-T has been approved for prostate cancer, there are other versions of antigen-presenting cellbased antigen-specific immunotherapies that are currently being developed by the Dendreon Corporation, such as lapuleucel-T, which targets human epidermal growth factor receptor 2 (Her-2/neu). Her-2/neu overexpression occurs in many epithelial cancers, and is commonly associated with more aggressive phenotypes. Like sipuleucel-T, this vaccine is generated by obtaining peripheral blood samples from patients with Her-2/neu positive tumors, which are then pulsed with a Her-2/neu-GM-CSF fusion protein (BA7072), and then reinfused back into patients. Two Phase I trials have been conducted with lapuleucel-T. The first trial was conducted in patients with metastatic, Her-2/neu-overexpressing breast cancer, and showed that patients developed T-cell proliferative immune responses to both the fusion protein and Her-2/neu alone, and interferon gamma enzyme-linked immunosorbent assay (ELISPOT) responses to the fusion protein. ${ }^{67}$ Furthermore, they found that four patients had clinical responses to the vaccine (one partial response and three cases of stable disease) ${ }^{67}$ The second trial was conducted in patients with metastatic breast, ovarian, or colon tumors which also expressed Her-2/neu. ${ }^{68}$ These studies found that patients receiving lapuleucel-T developed immune responses to the fusion protein, measured both by T-cell proliferative assays as well as interferon gamma ELISPOT. ${ }^{68}$ However, while both of these trials showed that the concept of sipuleucel-T can potentially be translated to other diseases, they also raise additional questions regarding the mechanism of action of these antigen-presenting cell-based vaccines, ie, whether the clinical effects are in fact in response to the activation of antigen-specific immune responses, or rather are due to the nonspecific immunostimulatory effects of the GM-CSF portion of the protein or the infusion of other immune cell types.

While sipuleucel-T was approved as a monotherapy for patients with asymptomatic-to-minimally-symptomatic, castrate-resistant metastatic disease, there are several possible future directions that could evaluate this therapy in other settings. For example, many studies have suggested that antitumor vaccines may have their greatest effect in the setting of minimal disease rather than bulky metastatic disease. Consequently, trials in patients with earlier-stage prostate cancer would seem advantageous, some of which are currently underway, including a trial being conducted in the neoadjuvant setting. ${ }^{69-71}$ In addition to studying different patient populations, another logical route of future investigation would be to investigate combinations of sipuleucel-T with other cancer therapies that could potentially provide a synergistic benefit. For example, a Phase II trial was conducted combining sipuleucel-T with bevacizumab, an antivascular endothelial growth factor antibody, based on previous investigations demonstrating that vascular endothelial growth factor production by tumors can inhibit the function of antigen-presenting cells. ${ }^{72}$ Combination treatment with sipuleucel-T and bevacizumab elicited a $>25 \%$ decrease in serum PSA in four of 22 patients, as well as an significant increase in median PSA doubling time and enhanced PA2024-specific immune responses. ${ }^{73}$ Other logical combination approaches might be to combine sipuleucel-T with approved prostate cancer therapies, such as androgen-deprivation therapy, a therapy with known immune-modulating activity, and newer androgen receptor antagonists or androgen biosynthesis inhibitors (such as abiraterone or MDV-3100), or undertake studies evaluating the appropriate timing/sequence when combined with chemotherapies. ${ }^{34,35}$

Alternatively, studies could be conducted combining sipuleucel-T with other immunomodulatory agents. For example, sipuleucel-T could be combined with antibodies targeting cytotoxic $\mathrm{T}$ lymphocyte antigen 4 , a surface molecule expressed on immunoregulatory cells and which also inhibits $\mathrm{T}$ cell proliferation; cytotoxic $\mathrm{T}$ lymphocyte antigen 4 blockade has been shown to act synergistically with other immunotherapeutic approaches. ${ }^{74-76}$ Finally, another logical approach might be to combine sipuleucel-T with other PAP-specific immunotherapies, which might provide a means to augment PAP-specific immune responses and enhance the clinical efficacy of these therapies. However, before combining sipuleucel- $\mathrm{T}$ with other immunotherapies, it may still be necessary first to define its mechanism of action to identify reliable markers that can be used to monitor the biological effect and/or clinical response.

Despite some of the ongoing challenges associated with implementing sipuleucel-T in clinical practice, there is clearly a major need for safe and effective therapies for patients with castrate-resistant metastatic disease. Indeed, this advanced stage of disease is rapidly developing into a very competitive market, with the development of abiraterone, MDV3100, and even other immunotherapies 
such as Prostvac. The availability of multiple therapies underscores the importance of identifying biomarkers which will predict benefit, so that the most appropriate therapies can be offered to enriched populations. In addition, rationally designed and rigorous sequence studies will be important to optimize survival and quality of life.

\section{Acknowledgments}

This work was supported for BMO and DGM by the National Institutes of Health (R01 CA142608) and the US Army Medical Research and Material Command.

\section{Disclosure}

$\mathrm{BMO}$ has no financial relationships or conflicts of interest to disclose. DGM has served as a consultant to Dendreon Corporation.

\section{References}

1. Jemal A, Siegel R, Ward E, Hao Y, Xu J, Thun MJ. Cancer statistics, 2009. CA Cancer J Clin. 2009;59(4):225-249.

2. Pound CR, Partin AW, Eisenberger MA, Chan DW, Pearson JD, Walsh PC. Natural history of progression after PSA elevation following radical prostatectomy. JAMA. 1999;281(17):1591-1597.

3. D'Amico AV, Schultz D, Loffredo M, et al. Biochemical outcome following external beam radiation therapy with or without androgen suppression therapy for clinically localized prostate cancer. JAMA. 2000;284(10):1280-1283.

4. Bianco FJ, Dotan ZA, Kattan MW, et al. Natural history of biochemically-recurrent castrate-resistant disease in men treated with maximal androgen blockage for a rising PSA after radical prostatectomy. Paper presented at the American Society of Clinical Oncology Prostate Cancer Symposium, February 17-19, 2005, Orlando, FL.

5. Burnstein KL. Regulation of androgen receptor levels: Implications for prostate cancer progression and therapy. J Cell Biochem. 2005;95(4): 657-669.

6. Heinlein CA, Chang C. Androgen receptor in prostate cancer. Endocr Rev. 2004;25(2):276-308.

7. Berry W, Dakhil S, Modiano M, Gregurich M, Asmar L. Phase III study of mitoxantrone plus low dose prednisone versus low dose prednisone alone in patients with asymptomatic hormone refractory prostate cancer. J Urol. 2002;168(6):2439-2443.

8. Ernst DS, Tannock IF, Winquist EW, et al. Randomized, doubleblind, controlled trial of mitoxantrone/prednisone and clodronate versus mitoxantrone/prednisone and placebo in patients with hormone-refractory prostate cancer and pain. J Clin Oncol. 2003;21(17): 3335-3342.

9. Kantoff PW, Halabi S, Conaway M, et al. Hydrocortisone with or without mitoxantrone in men with hormone-refractory prostate cancer: Results of the cancer and leukemia group B 9182 study. J Clin Oncol. 1999;17(8):2506-2513.

10. Petrylak DP, Tangen CM, Hussain MH, et al. Docetaxel and estramustine compared with mitoxantrone and prednisone for advanced refractory prostate cancer. $N$ Engl J Med. 2004;351(15): 1513-1520.

11. Tannock IF, de Wit R, Berry WR, et al. Docetaxel plus prednisone or mitoxantrone plus prednisone for advanced prostate cancer. $N$ Engl $J$ Med. 2004;351(15):1502-1512.
12. de Bono JS, Oudard S, Ozguroglu M, et al. Prednisone plus cabazitaxel or mitoxantrone for metastatic castration-resistant prostate cancer progressing after docetaxel treatment: A randomised open-label trial. Lancet. 2010;376(9747):1147-1154.

13. de Bono JS, Logothetis CJ, Fizazi K, et al. Abiraterone acetate plus low dose prednisone improves overall survival in patients with metastatic castration-resistant prostate cancer (CRPC) who have progressed after docetaxel-based chemotherapy: Results of COU-AA-301, a randomized double-blind placebo-controlled phase 3 study. Paper presented at the annual meeting of the European Society for Medical Oncology, April 28-May 1, 2010, Milan, Italy.

14. Ehrlich P. Über den jetzigen stand der karzinomforschung. Ned Tijdschr Geneeskd. 1909;5(1):273-290.

15. Thomas L. Discussion of cellular and humoral aspects of the hypersensitive states. In: Lawrence HS, editor. Cellular and Humoral Aspects of the Hypersensitive States. New York, NY: Hoeber-Harper; 1959.

16. Burnet FM. Immunological aspects of malignant disease. Lancet. 1967;1:1171.

17. Zarour H, DeLeo A, Finn O, Storkus W. Tumor antigens. In: HollandFrei Cancer Medicine. 6th ed. Hamilton, ON: BC Decker; 2003.

18. Hoeppner LH, Dubovsky JA, Dunphy EJ, McNeel DG. Humoral immune responses to testis antigens in sera from patients with prostate cancer. Cancer Immun. 2006;6:1.

19. Cheever MA, Allison JP, Ferris AS, et al. The prioritization of cancer antigens: A National Cancer Institute pilot project for the acceleration of translational research. Clin Cancer Res. 2009;15(17): 5323-5337.

20. Disis M, Calenoff E, McLaughlin G, et al. Existent T-cell and antibody immunity to HER-2/neu protein in patients with breast cancer. Cancer Res. 1994;54(1):16-20.

21. Hirohashi Y, Torigoe T, Inoda S, et al. The functioning antigens: Beyond just as the immunological targets. Cancer Sci. 2009;100(5): 798-806.

22. McNeel DG, Nguyen LD, Ellis WJ, Higano CS, Lange PH, Disis ML. Naturally occurring prostate cancer antigen-specific $\mathrm{T}$ cell responses of a Th1 phenotype can be detected in patients with prostate cancer. Prostate. 2001;47(3):222-229.

23. Challis GB, Stam HJ. The spontaneous regression of cancer. A review of cases from 1900 to 1987. Acta Oncol. 1990;29(5):545-550.

24. Pages F, Galon J, Dieu-Nosjean MC, Tartour E, Sautes-Fridman C, Fridman WH. Immune infiltration in human tumors: A prognostic factor that should not be ignored. Oncogene. 25;29(8):1093-1102.

25. Vesalainen S, Lipponen P, Talja M, Syrjanen K. Histological grade, perineural infiltration, tumour-infiltrating lymphocytes and apoptosis as determinants of long-term prognosis in prostatic adenocarcinoma. Eur J Cancer. 1994;30A(12):1797-1803.

26. Batstone GR, Doble A, Gaston JS. Autoimmune T cell responses to seminal plasma in chronic pelvic pain syndrome (CPPS). Clin Exp Immunol. 2002;128(2):302-307.

27. Alexander RB, Brady F, Ponniah S. Autoimmune prostatitis: Evidence of T cell reactivity with normal prostatic proteins. Urology. 1997; 50(6):893-899.

28. Palapattu GS, Sutcliffe S, Bastian PJ, et al. Prostate carcinogenesis and inflammation: Emerging insights. Carcinogenesis. 2005;26(7): 1170-1181.

29. McNeel DG, Nguyen LD, Disis ML. Identification of T helper epitopes from prostatic acid phosphatase. Cancer Res. 2001;61(13): 5161-5167.

30. Harada M, Matsueda S, Yao A, Ogata R, Noguchi M, Itoh K. Prostaterelated antigen-derived new peptides having the capacity of inducing prostate cancer-reactive CTLs in HLA-A2+ prostate cancer patients. Oncol Rep. 2004;12(3):601-607.

31. Olson BM, McNeel DG. Antibody and T-cell responses specific for the androgen receptor in patients with prostate cancer. Prostate. 2007;67(16):1729-1739. 
32. Saffran DC, Reiter RE, Jakobovits A, Witte ON. Target antigens for prostate cancer immunotherapy. Cancer Metastasis Rev. 1999;18(4): 437-449.

33. Aragon-Ching JB, Williams KM, Gulley JL. Impact of androgendeprivation therapy on the immune system: Implications for combination therapy of prostate cancer. Front Biosci. 2007;12:4957-4971.

34. Drake CG, Doody AD, Mihalyo MA, et al. Androgen ablation mitigates tolerance to a prostate/prostate cancer-restricted antigen. Cancer Cell. 2005;7(3):239-249.

35. Morse MD, McNeel DG. Prostate cancer patients on androgen deprivation therapy develop persistent changes in adaptive immune responses. Hum Immunol. 2010;71(5):496-504.

36. Roden AC, Moser MT, Tri SD, et al. Augmentation of T cell levels and responses induced by androgen deprivation. J Immunol. 2004;173(10): 6098-6108.

37. Noguchi M, Kakuma T, Uemura H, et al. A randomized phase II trial of personalized peptide vaccine plus low dose estramustine phosphate (EMP) versus standard dose EMP in patients with castration resistant prostate cancer. Cancer Immunol Immunother. 2010;59(7):1001-1009.

38. Karbach J, Neumann A, Atmaca A, et al. Efficient in vivo priming by vaccination with recombinant NY-ESO-1 protein and $\mathrm{CpG}$ in antigen naive prostate cancer patients. Clin Cancer Res. 2011;17(4):861-870.

39. Perambakam S, Hallmeyer S, Reddy S, et al. Induction of specific $\mathrm{T}$ cell immunity in patients with prostate cancer by vaccination with PSA146-154 peptide. Cancer Immunol Immunother. 2006;55(9): 1033-1042.

40. Fong L, Ruegg CL, Brockstedt D, Engleman EG, Laus R. Induction of tissue-specific autoimmune prostatitis with prostatic acid phosphatase immunization: Implications for immunotherapy of prostate cancer. J Immunol. 1997;159(7):3113-3117.

41. Pavlenko M, Roos AK, Lundqvist A, et al. A phase I trial of DNA vaccination with a plasmid expressing prostate-specific antigen in patients with hormone-refractory prostate cancer. Br J Cancer. 2004; 91(4):688-694.

42. McNeel DG, Dunphy EJ, Davies JG, et al. Safety and immunological efficacy of a DNA vaccine encoding prostatic acid phosphatase in patients with stage D0 prostate cancer. J Clin Oncol. 2009;27(25): 4047-4054.

43. Lubaroff DM, Konety BR, Link B, et al. Phase I clinical trial of an adenovirus/prostate-specific antigen vaccine for prostate cancer: Safety and immunologic results. Clin Cancer Res. 2009;15(23): 7375-7380.

44. Kantoff PW, Schuetz TJ, Blumenstein BA, et al. Overall survival analysis of a Phase II randomized controlled trial of a poxviral-based PSA-targeted immunotherapy in metastatic castration-resistant prostate cancer. J Clin Oncol. 2010;28(7):1099-1105.

45. Higano CS, Corman JM, Smith DC, et al. Phase 1/2 dose-escalation study of a GM-CSF-secreting, allogeneic, cellular immunotherapy for metastatic hormone-refractory prostate cancer. Cancer. 2008;113(5): 975-984.

46. Emens LA. GM-CSF-secreting vaccines for solid tumors. Curr Opin Investig Drugs. 2009;10(12):1315-1324.

47. Antonarakis ES, Drake CG. Current status of immunological therapies for prostate cancer. Curr Opin Urol. 2010;20(3):241-246.

48. Copier J, Dalgleish A. Whole-cell vaccines: A failure or a success waiting to happen? Curr Opin Mol Ther. 2010;12(1):14-20.

49. Murphy GP, Tjoa BA, Simmons SJ, et al. Phase II prostate cancer vaccine trial: Report of a study involving 37 patients with disease recurrence following primary treatment. Prostate. 1999;39(1):54-59.

50. Olson BM, Frye TP, Johnson LE, et al. HLA-A2-restricted T-cell epitopes specific for prostatic acid phosphatase. Cancer Immunol Immunother. 2010;59(6):943-953.

51. Becker J, Olson B, Johnson L, Davies J, Dunphy E, McNeel D. DNA vaccine encoding prostatic acid phosphatase (PAP) elicits long-term T-cell responses in patients with recurrent prostate cancer. J Immunother. 2010;33(6):639-647.
52. Machlenkin A, Azriel-Rosenfeld R, Volovitz I, et al. Preventive and therapeutic vaccination with PAP-3, a novel human prostate cancer peptide, inhibits carcinoma development in HLA transgenic mice. Cancer Immunol Immunother. 2007;56(2):217-226.

53. Wang Y, Harada M, Yano H, et al. Prostatic acid phosphatase as a target molecule in specific immunotherapy for patients with nonprostate adenocarcinoma. J Immunother. 2005;28(6):535-541.

54. Fong L, Brockstedt D, Benike C, et al. Dendritic cell-based xenoantigen vaccination for prostate cancer immunotherapy. J Immunol. 2001;167(12):7150-7156.

55. Fong L, Ruegg CL, Brockstedt D, Engleman EG, Laus R. Induction of tissue-specific autoimmune prostatitis with prostatic acid phosphatase immunization: Implications for immunotherapy of prostate cancer. J Immunol. 1997;159(7):3113-3117.

56. US Food and Drug Administration labeling information - Provenge. 2010. Available at: http://www.fda.gov/downloads/BiologicsBlood Vaccines/CellularGeneTherapyProducts/Approved Products/ UCM210031.pdf. Accessed April 1, 2011.

57. Laus R, Yang D, Ruegg C, et al. Dendritic cell immunotherapy of prostate cancer: Preclinical models and early clinical experience. Cancer Res Ther Control. 2001;11:1-10.

58. Small EJ, Fratesi P, Reese DM, et al. Immunotherapy of hormonerefractory prostate cancer with antigen-loaded dendritic cells. J Clin Oncol. 2000;18(23):3894-3903.

59. Takaue Y, Tanosaki R, Tobisu K, et al. Antigen-pulsed dendritic cell therapy for the treatment of hormone-refractory prostate cancer: A phase I trial of APC8015. Paper presented at the 38th annual meeting of the American Society of Clinical Oncology, May 18-21, 2002, Orlando, FL.

60. Burch PA, Breen JK, Buckner JC, et al. Priming tissue-specific cellular immunity in a phase I trial of autologous dendritic cells for prostate cancer. Clin Cancer Res. 2000;6(6):2175-2182.

61. Burch P, Croghan G, Gastineau D, et al. Immunotherapy (APC8015, Provenge) targeting prostatic acid phosphatase can induce durable remission of metastatic androgen-independent prostate cancer: A Phase II trial. Prostate. 2004;60(3):197-204.

62. Small EJ, Schellhammer PF, Higano CS, et al. Placebo-controlled phase III trial of immunologic therapy with sipuleucel-T (APC8015) in patients with metastatic, asymptomatic hormone refractory prostate cancer. J Clin Oncol. 2006;24(19):3089-3094.

63. Higano CS, Schellhammer PF, Small EJ, et al. Integrated data from 2 randomized, double-blind, placebo-controlled, phase 3 trials of active cellular immunotherapy with sipuleucel-T in advanced prostate cancer. Cancer. 2009;115(16):3670-3679.

64. Kantoff PW, Higano CS, Shore ND, et al. Sipuleucel-T immunotherapy for castration-resistant prostate cancer. $N$ Engl J Med. 2010;363(5): $411-422$.

65. Silverman E. Provenge approval means sensitive coverage decisions. Manage Care. 2010;19(9):31-34.

66. Longo DL. New therapies for castration-resistant prostate cancer. $N$ Engl J Med. 2010;363(5):479-481.

67. Park JW, Melisko ME, Esserman LJ, Jones LA, Wollan JB, Sims R. Treatment with autologous antigen-presenting cells activated with the HER-2 based antigen Lapuleucel-T: Results of a phase I study in immunologic and clinical activity in HER-2 overexpressing breast cancer. J Clin Oncol. 2007;25(24):3680-3687.

68. Peethambaram PP, Melisko ME, Rinn KJ, et al. A phase I trial of immunotherapy with lapuleucel-T (APC8024) in patients with refractory metastatic tumors that express HER-2/neu. Clin Cancer Res. 2009; 15(18):5937-5944.

69. Brichard VG, Lejeune D. Cancer immunotherapy targeting tumourspecific antigens: Towards a new therapy for minimal residual disease. Exp Opin Biol Ther. 2008;8(7):951-968.

70. Gray A, Raff AB, Chiriva-Internati M, Chen SY, Kast WM. A paradigm shift in therapeutic vaccination of cancer patients: The need to apply therapeutic vaccination strategies in the preventive setting. Immunol Rev. 2008;222:316-327. 
71. Gray A, Garcia-Hernandez MD, van West M, Kanodia S, Hubby B, Kast WM. Prostate cancer immunotherapy yields superior long-term survival in TRAMP mice when administered at an early stage of carcinogenesis prior to the establishment of tumor-associated immunosuppression at later stages. Vaccine. 2009;27:G52-G59.

72. Gabrilovich DI, Chen HL, Girgis KR, et al. Production of vascular endothelial growth factor by human tumors inhibits the functional maturation of dendritic cells. Nat Med. 1996;2(10):1096-1103.

73. Rini BI, Weinberg V, Fong L, Conry S, Hershberg RM, Small EJ. Combination immunotherapy with prostatic acid phosphatase pulsed antigen-presenting cells (provenge) plus bevacizumab in patients with serologic progression of prostate cancer after definitive local therapy. Cancer. 2006;107(1):67-74.
74. Gregor PD, Wolchok JD, Ferrone CR, et al. CTLA-4 blockade in combination with xenogeneic DNA vaccines enhances T-cell responses, tumor immunity and autoimmunity to self antigens in animal and cellular model systems. Vaccine. 2004;22(13-14):1700-1708.

75. Sarnaik AA, Yu B, Yu D, et al. Extended dose ipilimumab with a peptide vaccine: Immune correlates associated with clinical benefit in patients with resected high-risk stage IIIc/IV melanoma. Clin Cancer Res. 2011;17(4):896-906.

76. Hurwitz AA, Foster BA, Kwon ED, et al. Combination immunotherapy of primary prostate cancer in a transgenic mouse model using CTLA-4 blockade. Cancer Res. 2000;60(9):2444-2448.

\section{Publish your work in this journal}

The Open Access Journal of Urology is an international, peer-reviewed, open access journal publishing original research, reports, editorials, reviews and commentaries on all aspects of adult and pediatric urology in the clinic and laboratory including the following topics: Pathology, pathophysiology of urological disease; Investigation and treatment of

\section{Dovepress}

urological disease; Pharmacology of drugs used for the treatment of urological disease. The manuscript management system is completely online and includes a very quick and fair peer-review system, which is all easy to use. Visit http://www.dovepress.com/testimonials.php to read real quotes from published authors.

Submit your manuscript here: http://www.dovepress.com/open-access-journal-of-urology-journal 